\title{
Le printemps sacrifié
}

\section{Gilbert Durand}

\section{(2) OpenEdition \\ Journals}

Édition électronique

URL : http://journals.openedition.org/shakespeare/1303

DOI : $10.4000 /$ shakespeare. 1303

ISSN : 2271-6424

Éditeur

Société Française Shakespeare

Édition imprimée

Date de publication : 1 novembre 1995

Pagination : 135-155

Référence électronique

Gilbert Durand, «Le printemps sacrifié », Actes des congrès de la Société française Shakespeare [En ligne], 13 | 1995, mis en ligne le 01 janvier 2007, consulté le 21 avril 2019. URL : http:// journals.openedition.org/shakespeare/1303 ; DOI : 10.4000/shakespeare.1303 


\title{
SHAKESPEARE \\ LE MONDE VERT : RITES ET RENOUVEAU
}

Société Française Shakespeare

\author{
Actes du Congrès 1994 \\ sous la direction \\ de \\ M.T. JONES-DAVIES
}

Ouvrage publié avec le soutien de

BARCLAYS

BARCLAYS BANK S.A.

PARIS

LES BELLES LETTRES

1995 
Tous droits de traduction, de reproduction et d'adaptation réservés pour tous les pays.
(C) 1995 Société d'édition Les Belles Lettres, 95 bd Raspail 75006 Paris 


\section{Du Complexe d'Ophélie au Mythe du Printemps sacrifié}

$\grave{a ̀ ~ J e a n ~ P e r r i n ~}$

Lorsque Madame M. T. Jones Davies m'invita aimablement à participer à ce colloque - au si beau titre inspiré par une intuition de Northrop Frye sur le "monde vert" shakespearien - mon premier mouvement fut de refuser cet honneur, car je me sens trop infirme en anglophonie (au moins autant que Jean François Ducis qui, en 1770, fut le premier "adaptateur" en langue française de l'œuvre du grand dramaturge) et ne suis qu'un spectateur de Shakespeare, bien plus même: un amateur passionné de théâtre lyrique. Et là combien ai je encore rencontré Shakespeare de Purcell à Verdi ... Je ne sais que trop, avec André Gidel, quels sont les pièges de la traduction, donc de l'interprétation. Gide nous dit quelles difficultés il y a de traduire le mot mallard (Antoine et Cléopâtre III.10) en français où tout "canard" est soit boiteux, soit enchaîné. Au plus chaud de la bataille d'Actium, Gide propose de remplacer ce "canard" par le baudelairien "albatros". Mais l'illustre traducteur, qui connaît cependant le "mallart" (canard sauvage mâle) du patois solognot, perd toute la saveur sémantique et cynégétique de ce mot, oubliant que le "mallart" suit toujours dans son vol la cane, comme le navire d'Antoine suit celui de Cléopâtre en fuite ...

Mon premier mouvement "bis" se laissa entraîner par l'image d'Ophélie suscitant en moi l'appellation de "printemps sacrifié". Image du beau tableau de John Everett Millais (1852) et des dessins obsédants de 1854 : The lady of Shallot (que je n'ose traduire, tant le terme est culinaire en français par "la dame a l'échalote"). Image reprise par Rimbaud "la blanche Ophélie comme un grand lys ...", par Jules Laforgue : "Ophélie, Ophélie ton beau corps sur l'étang ...", par Saint Pol-Roux : "un jour mon âme se jeta dans la rivière des Ophélies ...", par Joachim Gasquet qui, justement, unit la vision de la "triste Ophélie" a celle du printemps: "un moment au-dessus de l'eau, je vis encore ses pieds aussi purs, aussi immatériels que ceux de la Primavera ...". Pour nous aussi, réunis ici afin de commémorer Rites et Renou- 
veau a l'époque élisabethaine, l'image du préraphaëlite ... appelle dans notre mémoire celle apparue a Botticelli.

Mais qu'est-ce à dire lorsqu'on prononce le beau mot de "Printemps"? Il faut écarter les fleurettes trop faciles, ces "filles -fleurs" réductrices, qui bloquent de leur enchantement le symbolisme des Saisons. Le Printemps c'est d'abord le triomphe de la vie sur le néant de l'hiver, face au déclin de l'automne. C'est aussi la pureté virginale, la "promesse des fleurs" avant les fructifications de l'été ... C'est bien la Primavera, qui selon Pierre Somvile ${ }^{2}$ est l'exact pendant de la Naissance de Vénus, mais rien n'est sacrifié chez ces hiératiques et douces images de Botticelli, verticales ou prenant leur envol ; on ne peut en dire autant de la triste Ophélie couchée dans les eaux sombres et que la folie pousse à la mort aquatique. Là le printemps est sacrifié, et le sacrifice tout à coup "interdit toute espérance", interdit l'accès à la plénitude de l'être ... Et j'étais très content, d'entendre hier, de la bouche même d'un éminent seiziémiste, mon ami Claude G. Dubois ce constat comme quoi au siècle de Shakespeare l'envers du Printemps, la mélancolie rôde toujours dans les coulisses. Primavera rime avec Melancolia ... C'est qu'un bon siècle s'est écoulé entre le printanier quattrocento du florentin, et le règne d'Elisabeth en Angleterre. Et quel siècle que celui qui porte la déchirure définitive de la Chrétienté, les Guerres de Religions, l'Europe mise à feu et à sang par Charles Quint, la conquête du Nouveau Monde par tant d'armadas, la mort tragique d'Henri Il de France, l'assassinat d'Henri III, l'avènement des Tudors et l'exécution par Henri VIII de sa femme, la mère de la reine Elisabeth ... et cette dernière faisant mettre à mort Marie Stuart! Siècle qui décapite Thomas More, siècle de Rabelais certes, mais aussi de Pichrocole, de Montaigne, de Shakespeare et aussi de Nostradamus et de Cornélius Agrippa ... Les fruits sanglants du XVI e siècle ne "passent pas" la promesse des fleurs du quattrocento! "Sombre et scélérat XVI" siècle", comme l'écrit Hugo.

Le "premier mouvement" de notre imaginaire, attendri par l'image d'Ophélie, contrairement à ce qu'affirme Talleyrand de tout "premier mouvement", ne fut pas le bon, tout au moins le bon pour donner de l'étoffe dramatique (au sens étymologique de drama action) à un mythe. Comme l'a bien vu Bachelard ${ }^{3}$, le beau nom d'Ophélie couvre tout au plus un "complexe": le complexe d'images qui relient, sans cohérence dramatique la féminité/l'eau/la mort/les larmes, un mythe indéterminé, comme celui du Prin- 
temps sacrifié, ou bien celui d'Aréthuse que Jean Perrin ${ }^{4}$, jadis, décela bellement chez Shelley, ne vient qu'après. Comme tout "complexe", celui de Caron (G.Bachelard) celui, bien entendu, d'Edipe ou de Jocaste, il se dénomme facilement, mais perd beaucoup de sa discursivité dramatique. Il a un état civil trop précis, si je puis dire, au contraire de la geste ou du drame, que retrace tout sermo mythicus, tout mythe. Très souvent le mythe a des difficultés à trouver un nom - pour des raisons que la raison ne connaît pas et sur lesquelles nous ne pouvons nous arrêter ici - et, comme le remarque profondément Roger Bastide à propos d'André Gide $^{5}$, il demeure latent, mal dicible, soit que le nom du héros mythique se réduise a un attribut souvent épithétique, ("colère d'Héra", c'est-à-dire Héraclès ; "fuyante" c'est-à-dire "Agar", "Stator", "Tonnans", "Anxurus", "Optimus" c'est-à-dire Jupiter, "Argeiphontes" c'est-à-dire Hermès, etc.), soit qu'il se cherche désespérément comme chez Gide - à travers Edipe, Colomb, ou Saül, soit encore que l'indécision du nom porte à quelque méprise, à ce que nous appelons un "travesti" et dont on trouve maints exemples dans l'hagiographie populaire. Il faut préciser que cette imprécision dans la dénomination vient plus profondément - Ricœur l'a fort bien montré, ce qui est corroboré en partie par la théorie de la "réception" - du "conflit des interprétations" : réduire Edipe au "complexe" de la haine du père, voire de l'attrait pour la mère, c'est oublier Edipe Roi et ne retenir qu'une image dans le mythe. Pour le mythicien, et la mythocritique qu'il applique, le mythe ne commence pas par un nom de baptême, mais par une structure mythogénique, et - j'insiste bien sur ce point! - par une "structure figurative", c'est-à-dire qui ne se contente pas du petit jeu binaire des structuralismes, mais fait appel aux contenus symboliques, et spécialement aux "mythèmes" (Cl. Lévi-Strauss) ${ }^{6}$ que signale, dans le sermo, leur redondance. Ces mythèmes sont souvent des attributs, des épithètes ou une action verbale, secondairement un seul nom propre.

Dans son étude passionnée de William Shakespeare, Victor $\mathrm{Hugo}^{7}$ nous donne un bel exemple de la découverte de ce "fait fort étrange", de ces redondances qu'il repère dans "trente quatre sur trente six" (sic) des pièces du dramaturge anglais. ll observe, sauf dans Macbeth et dans Romeo, une "double action qui traverse le drame et qui le reflète en petit". Ce dédoublement - plus loin Hugo ira jusqu'au "triplement" - est bien l'ébauche de la redondance signalétique du mythème qui constitue le mythe. Notre grand 
poète serait donc, à son insu, le véritable créateur de notre moderne mythocritique! Ainsi, écrit-il,

"Hamlet fait au-dessous de lui, un Hamlet. Il tue Polonius père de Laërtes, et voila Laërtes vis a vis de lui dans la même situation que lui vis à vis de Claudius ..."

Même redondance repérée dans le Roi Lear, où le vieux roi désespéré par ses deux filles et consolé par la troisième Cordélia, se voit doublé,

"et répété par Glocester, trahi par son fils Edmond et aimé par son fils Edgar. ... un drame moindre copiant et coudoyant le drame principal, l'action traînant sa lune, une action plus petite sa pareille ..."

Peu importe le nom propre, c'est la fonction attributive, l'action qu'indique le verbe qui compte ! Et selon la vue profonde du grand poète, tout mythe est bien une constellation où se hiérarchisent lunes et planètes homologues.

Donc l'essentiel d'Ophélie n'est pas dans son "complexe" : un complexe peut cacher un train mythique ! Il est, somme toute, un cliché un peu figé. Peut-on passer de ce "complexe" de la jeune morte, couronnée de fleurs printanières, "pâquerettes, coucous, lamiers, pattes de loup ...", pleurée par toute la Cour de Danemark, à la rhétorique (sermo) ou au "drame" (drama) d'un mythe ? Il semble que oui si l'on examine la situation dramatique de l'infortunée fillette. Car l'abandon de la vie d'Ophélie à l'eau de la rivière, s'enchaîne à un autre abandon : celui perpétré par Hamlet à l'acte III (sc.1) "Si bas, si bas tombé", qui renie sa promesse faite à Ophélie et en tient des propos blessants :

" ... la Beauté aura pouvoir de réduire l'honnêteté en ma querelle ... Au couvent ! entre au couvent !. ... je ne t'aimais pas ..."

Et à cette folie feinte par ruse du jeune Prince, s'ajoute à la scène 4 le terrible quiproquo, lorsque le père d'Ophélie, Polonius, caché par une tenture est transpercé par l'épée d'Hamlet croyant sacrifier l'usurpateur criminel Claudius. Voilà bien de quoi tisser un mythe ! Voilà bien un double "drame" : le parjure de l'amant et l'assassinat du père, qui conduit Ophélie à perdre l'esprit pour de bon quant à elle, à perdre la vie dans la rivière, ce qui conduira à un tiers drame dans ce parallélisme qu'avait décelé Hugo : le frère d'Ophélie Laërtes chargé d'une double vengeance, et surtout de celle de son père Polonius et cherchant la mort du meurtrier ... Le tou- 
chant "complexe d'Ophélie" cache, à vrai dire, toute une série d'enchaînements mythiques parce que signés de la redondance. Le tendre "Printemps sacrifié" cache le sombre mythologème de l'éternel hiver de l'erreur, du mensonge, de la falsification du réel ... Tout mythe, par rapport à une simple image fut-elle "obsédante" est toujours une chaîne, un discours étiologique. Cl. Lévi-Strauss l'a bien vu lorsqu'il dit que le sermo mythicus est une tentative pour résoudre un dilemme ${ }^{8}$, une énigme. Ajoutons qu'il est une tentative non logique, non naturaliste, à établir un panorama des solutions. Nous renvoyons aux définitions du mythe que nous avons proposées ailleurs.

Qu'est-ce à dire plus profondément? Que c'est le dessein qu'a choisi Hamlet - dans sa vengeance implacable contre le Roi usurpateur Claudius - qui mène le double quiproquo mortel : feindre la folie devant Ophélie, percer la tenture trompeuse cachant Polonius. La folie d'Hamlet est choisie, comme celle de Brutus, comme celle d'Hugolin ou celle d'Héraclien de Nisbie - selon Hugo p. 197- ou celle que conseillait Océanos à Prométhée, comme plus tard dans le Roi Lear :

"la folie feinte d'Edgar qui éclaire la folie feinte d'Hamlet ..."

Donc la gracieuse et touchante scène d'Ophélie abandonnée dans les eaux, qu'ont chantée tant de poètes, qu'ont illustrée bien des peintres, repose sur un fondement mythique : celui du Printemps sacrifié par un implacable enchaînement. L'accent ne porte plus sur l'image de la Primavera, mais sur le récit qui expose les raisons - ou les déraisons ! - du sacrifice.

Or ce mythologème (c'est-à- dire, pour faire bref, ce scénario abstrait d'un mythe) de la jeune innocente, du Printemps de la vie sacrifié foisonne bien en deçà et au-delà de la fiancée bafouée et blessée d'Hamlet.

C'est, bien sûr, celui d'Iphigénie - illustré, depuis Euripide, et par Racine, et par Goethe - c'est celui si touchant de la fille de Jephté, (qu'explicitement Hamlet assimilera à Ophélie lorsqu' il demande à Polonius - Acte II sc.2 - "que t'en semble mon vieux Jephté ?") c'est aussi celui de la "veuve" Didon, comme l'appelle plaisamment Gonzalo a l'acte Il (sc. 1) de $L a$ Tempête, et c'est celui d'Iseut/Isolde ... Mais bien au-delà des traditions grecque et biblique, dans l'immense littérature chinoise ${ }^{9}$, on retrouve le mythe du printemps sacrifié, soit dans le recueil de contes du XVI ${ }^{\mathrm{e}}$ siècle, Du Shiniang la prostituée fidèle, trompée et 
revendue à un riche marchand par son amant, et qui se jette après avoir jeté ses bijoux ... et non les avoir rendus à Hamlet :

"Monseigneur, j'ai tenu de vous quelques souvenirs qu'il me tardait beaucoup de vous rendre ..." (Hamlet III.1) dans le fleuve. L'héroïne du grand roman classique du XVIIle siècle, tant repris par l'Opéra de Pékin, Le rêve dans le Pavillon rouge où Lin Daiyu meurt de chagrin d'amour, malade et abandonnée ... c'est déjà la Dame aux Camélias, c'est déjà La Traviata, c'est déjà, peut-être, Eugénie Grandet, c'est encore la légende chinoise Zhu Ying taï "amour des papillons" où l'on voit deux amants morts de mélancolie transformés en papillons immortels après leur mort ... c'est déjà Roméo et Juliette. Mythologème d'une banalité extrême, portant mille noms selon les époques, selon les civilisations, plus passible d'une mythanalyse que d'une mythocritique précise.

C'est cependant chez Shakespeare que ce scénario devient mythe en se signalant, à la fois avec une obsédante redondance, et à la fois avec un trait (un mythème) très distinctif. L'on assiste même à un abandon du complexe limité d'Ophélie - car les noyades seront bien rares, reléguées par le poignard, le poison ou la strangulation - au profit d'un mythe plus ample, constitué de mythèmes plus nombreux et qui prend plusieurs noms au cours de la dramaturgie shakespearienne. Ophélie n'étant qu'un cas particulier, vite rejoint (pour nous, non pour la stricte chronologie de la création) par le cortège de Lucrèce, de Thysbé, de Juliette, d'Hermione, d'Imogène, de Desdémone et même de Cléopâtre. Ces redondances - ou comme dit Mauron ces "obsessions" - qui signent un mythe ne lâchent plus le poète depuis le poème de 1594 Le viol de Lucrèce et la Comédie des erreurs de 1593. L'un sera la source de tout le tragique, l'autre de toute la profondeur philosophique - cette "mâle gaîté" qu'un autre poète décelera chez Molière - des comédies. Il faut auparavant noter que dans le mythologème banal des vierges sacrifiées dont Iphigénie est le paradigme, c'est le destin, le poids du fatum ou des Parques qui noue - ou dénoue ! - la vie de la frêle et innocente victime. Et le janséniste Racine sera, oh combien, sensible à ces prédestinations ... Ce destin, celui que filent les nornes est symbolisé chez Wagner par les philtres. Mais dans ces ultimes années de la fin du XVIe siècle, s'affirme encore ce que J. Burckart après Stendhal ${ }^{10}$, privilégiera dans le déploiement de la Renaissance : l'émergence de l'in- 
dividu et de ses responsabilités, l'émergence de la virtu. Le sacrifice du Printemps juvénile ne viendra plus des dieux, mais du choix de l'homme dans ses désirs et dans ses volontés. C'est là qu'est l'originalité d'un Pantagruel, d'un Montaigne, d'un Shakespeare.

Nous pouvons relever trois phases dans le déroulement de l'œuvre du dramaturge, trois phases qui correspondent à trois motifs, ou plus fortement trois causes qui déterminent le saccage et le sacrifice du Printemps ... Comme l'ont bien noté Cl. G. Dubois et les spécialistes du Baroque, l'art si exubérant du XVI e siècle finissant s'imprègne et s'obsède des images de la mort ... Pour Dubois le "Que sais-je ?" de Montaigne est bien vite suivi du "Qu'est-ce qu'être ?". "Le verbe être ne se pense qu'en fonction de son antonyme qui prend ... (alors) la forme de mourir"11. Pour Dominique Fernandez, dans sa belle étude Le Banquet des Anges, la mort - ici la peste - "explique en partie l'épanouissement du style baroque" parce qu'est rendu sensible par là "l'atroce labilité de la condition humaine". Le Baroque s'oppose donc au "monde carré et solide de Raphaël", "au classicisme de la Renaissance"12. Ajoutons : "à la Primavera de Botticelli". Nous allons successivement survoler ces trois prises de responsabilité du sacrificateur dans l'œuvre de Shakespeare :

- Dans un premier temps, dans le sillage du Viol de Lucrèce et de la Comédie des Erreurs, la responsabilité du sacrifice tombe sur celui qui se trompe parce qu'il suit trop vite l'apparence.

- Dans un second temps, plus profondément, l'apparence va susciter, comme une sorte de relais causal qui armera le sacrificateur : la jalousie.

- Dans un troisième temps enfin, qui correspond aux ultimes pièces du poète, 5 ou 6 ans après la mort d'Elisabeth (1603), l'extinction des Tudors et l'extraordinaire succession au trône de Jacques 1er (le fils même de celle que la reine avait envoyée a l'échafaud), la noirceur et le froid de l'hiver "tueur de pauvres" jeunes gens sont sublimés par les miracles d'un Conte d'hiver où le romanesque le plus fantastique reprend le dessus.

Le Viol de Lucrèce déploré est bien le "prélude prolixe aux grandes tragédies" comme l'écrit mon ami Henri Fluchère, emprunté à l'Ovide des Fastes (721-852) et à l'Histoire romaine de Tite-Live (I, 57-69). C'est la mise en place de la constante éthique de l'ordre, ici représenté par la fidélité, cette virginité éternelle de la 
femme fidèle, son printemps éternel qui résonnera encore dans le viol de Lavinia du Titus Andronicus. Printemps "saccagé" comme dit encore Fluchêre - saccagé jusqu'au suicide de la vierge violée par le "villain" Tarquin. L'infortunée Lucrèce tente de calmer, ou du moins comparer, son irrémédiable infortune en contemplant un tableau de la Chute de Troie représentant Hécube sacrifiée par le féroce Pyrrhus ; il est bien remarquable que cette scène "redoublant" la douleur de Lucrèce, soit encore redondante à l'acte II.2 de Hamlet dans la pièce (encore théâtre dans le théâtre, après celui du Songe !) que viennent jouer les comédiens à Hamlet et Polonius. Le prestigieux traducteur, André Gide n'a pas compris la signification de cet intermède et avoue, dans une note, avoir "simplifié grandement le texte", or non seulement au premier degré cette scène typifie l'horreur de Troie sacrifiée, mais encore par le personnage de Pyrrhus elle rime sémantiquement avec la situation de l'usurpateur du Danemark, Claudius, ayant épousé la femme du roi qu'il a tué ... Cette "redondance" n' est donc point innocente! C'est par cette image obsédante qui relie le vieux mythe de l'Iliade à l'Histoire romaine et finalement a la tragédie du Prince de Danemark que s'instaure une éthique fondamentale et pérenne dans toute l'œuvre de Shakespeare qui nous fait oublier le XVIe siècle et nous touche si profondément. C'est par cette universalité signalée par les redondances constitutives du mythe, que l'œuvre du poète élisabéthain - selon un beau mot d'Henry Corbin — nous "regarde". Cette obsession de l'ordre et la crainte devant le désordre sera le moteur de toute la dramaturgie de Shakespeare, depuis les drames "historiques" jusqu' aux ultimes tragédies "rédemptrices" des années 1605,1610 ... Et en lisant la pièce Henri VI (Ière séquence) on s'en vient a regretter que le grand poète de la virginité fut anglais, donc ne vit en Jeanne d'Arc qu'une "sorcière" face à l'héroïque Talbot, car pour nous Français la Pucelle illustre totalement le mythème de la virginité trahie, bafouée, sacrifiée. Il n'est pas indifférent de noter avec la savante Frances A. Yates ${ }^{13}$ combien ce thème de "l'ordre virginal" — si je puis dire - est lié étroitement à l'apologie d'Elisabeth Ière, la reine vierge, la virgo, l'Astraea. Belle illustration de la concordance de nos mythanalyses et de "l'esthétique de la réception" de notre collègue de Konstanz H. R. Jauss ${ }^{14}$ ! Celui-ci me dit souvent que nos deux procédures méthodologiques sont, certes inverses, mais "complémentaires" et cela se vérifie ici, dans ce XVI ${ }^{e}$ siècle anglais finissant, où l'on voit la banale éthique de l'ordre et la banale image de la vierge, 
renforcées par leur actualisation élisabéthaine. A cette première apparition de notre mythe dans le Viol de Lucrèce, il faut joindre la Comédie des Erreurs laquelle va pour ainsi dire compléter la philosophie du viol de l'ordre, du sacrifice de l'être. La Comédie des Erreurs nous donne la clef de tout désordre : c'est l'Erreur qui guette les personnages de toute Comédie Humaine, elle opère les substitutions, elle construit les apparences, inaugure le quiproquo. Quelques années plus tard - 1598 ?- une "seconde Comédie des Erreurs" (selon Fluchère) viendra renforcer ce thème, de la nocivité de la falsification par l'apparence, et finalement du triomphe de l'identité, du face à face réel en Béatrice et Bénédict de Beaucoup de bruit pour rien, au titre si amer et si désabusé. Enfin en 1599 , Jules César confirme cette dénonciation de l'erreur lorsqu' au V acte, Messala, l'ami de Brutus, s'écrie :

"O erreur haïssable, toujours prête à montrer à la pensée des hommes des choses qui ne sont point !“

Dans les autres comédies des années 1590-1595, les mêmes redondances obsédantes se décèlent : dans la Mégère apprivoisée (explicitée dans le Prologue), c'est Christophe Sly, le chaudronnier ivrogne, qui se voit dépouillé illico de son identité et devient lord tandis qu'un valet en travesti se dit sa femme! Toute la pièce tourne autour de cette identité bafouée dès le prologue, tout y est travesti : Petruchio et Catharina sont deux personnalités postiches qui ignorent leur identité jusqu' au dénouement final où l'éthique du "même" reprend ses droits contre les altérations, les altérités de l'autre ... Dans les Deux Gentilshommes de Vérone c'est le dédoublement généralisé de tous les personnages : deux amoureux, deux galants, deux pères, deux pages, et tant de dédoublements introduisent un vertige d'identification ... Mais dès le Marchand de Venise et le Songe d'une nuit d'été, ce qui n'était qu'images de situations obsédantes, angoisses devant la perte d'identité vite camouflée par le rire, va prendre soudain sa dimension mythique qu'elle n'abandonnera jamais au cours de toutes les grandes œuvres à venir. Prendre sa dimension «manifestée" — comme dirait Roger Bastide ou Sigmund Freud - après bien des latences. D'abord en se donnant une sorte d'emblème paradigmatique dans le Marchand de Venise, ensuite - sans que cet "ensuite" ne soit chronologique puisque la chronologie de ces comédies reste flottante à deux ou trois années près : $1594,1595,1596 \ldots$ - dans le Songe en 
prenant son nom mythique, moment où pour nous le complexe d'Ophélie se résorbe en mythe de Thysbé.

L'emblème des coffrets, dans le Marchand de Venise, constitue bien ce que nous appelons - Lévi-Strauss et moi - dans notre jargon de mythiciens, un "mythème", c'est-à-dire la plus petite unité de sens dans un récit, unité qui peut être une action, une situation, un objet emblème et qui signale son importance par la redondance, le jeu des similitudes, des homologies ... Et c'est ce dernier cas ici : c'est la série des coffrets (acte II, sc. 7) que la riche Portia présente aux prétendants. L'un d'or porte cette inscription : "Qui me choisit gagnera ce que bien des hommes désirent", le second d'argent: "Qui me choisit obtiendra ce qu'il mérite", enfin le troisième de "plomb grossier" : "Qui me choisit doit donner et hasarder tout ce qu'il a". Le Prince marocain choisit le ler et n'y trouve qu'un squelette: "tout ce qui brille n'est pas d'or" ! Le Prince d'Aragon (Acte II. sc. 9) ouvre le coffret d'argent et y trouve le portrait d'un idiot, enfin Bassano (III. sc. 2) choisit ce coffret de plomb "qui fait une menace plutôt qu'une promesse", qui contient le portrait de la belle Portia et la devise, devise qui peut servir à toute vertu chez Shakespeare: "A vous qui ne choisissez pas sur l'apparence ..." L'on sait comment la comédie se poursuit et se conclut à travers un imbroglio et des travestissements invraisemblables, des parallélismes de destins couplés Bassano/Portia, Nerissa/Gratiano, où l'identité découverte dans le coffret de plomb est sans cesse menacée par de doubles dédoublements :

"Remarquez bien ça, dit Portia de Bassano, il se voit double dans mes deux yeux, une fois dans chaque oeil, donnez votre parole d'homme double : voilà un serment qui mérite crédit !!“

mais finalement l'unité identificatrice triomphe puisque le docteur n'était que Bassano et le clerc du docteur n'est autre que Gratiano ... Ne jamais choisir sur l'apparence ... être sans se poser la fameuse question puisqu' il faut "donner et hasarder" tout ce qu'on $a$... Ethique des coffrets bien établie à laquelle failliront la plupart des héros de Shakespeare: Roméo, Juliette, Ophélie, Hamlet, Othello ... Tous vont "choisir" sur l'apparence, et c'est ce que dit le mythe de Pyrame, le mythe de Roméo, le mythe d'Hamlet ... Nul n'est besoin de décrire, ici, en détail le fameux mythe que Shakespeare emprunte à Ovide, exemple classique de redoublement d'un "théâtre dans le théâtre", d'une "mise en abîme" à l'Acte V du Songe, et où tous les "mythèmes" du drame shakespearien sont 
stylisés et mis a plat par les rustres bouffons : les amants séparés (par le mur) - la tombe de Ninus - le voile trompeur ensanglanté d'un sang trompeur - la mort d'un premier héros trompé et "printanier" :

"... ces lèvres de lys, ce nez cerise, ces joues jaunes comme primevére ... ces yeux verts comme des poireaux" -

le fatal enchaînement du second héros trompé par la mort abusée $\mathrm{du}$ premier. Ces cinq mythèmes (outre un sixième qui est la clef de tous les autres : la vierge, la fidélité fragilisées) : la séparation - la tombe - l'objet trompeur - la première conséquence funeste de l'erreur - la seconde conséquenc qui s'enchaîne, vont se retrouver partiellement ou totalement dans toute la dramaturgie shakespearienne : et la même année où paraît ce Songe d'une nuit d'été (qui est curieusement situé la veille de la Saint Valentin!) qui, par ailleurs, est un hymne agreste au Printemps, paraît Roméo et Juliette qui répond mythème par mythème aux cinq articulations du mythe de Pyrame. Une remarque peut être introduite ici : dans le Songe, à part l'acte I et l'acte V, encore que ce dernier transporte, par la comédie des artisans, la nature dans Athènes, toutes les scènes de l'acte $(2,3,4)$ se déroulent dans la nature comme la meilleure partie de Comme il vous plaira se situe dans la Forêt des Ardennes ... Sans aller jusqu'à affirmer que le grand Bill était déjà écologiste ou pour le moins rousseauiste, on peut remarquer que Shakespeare situe toute tromperie dans la ville : Ephèse (Comédie des Erreurs), Venise (Le Marchand), Messine / Padoue (La Mégère), Vérone (Les deux Gentilshommes), Messine (Beaucoup de bruit), Milan et, bien sûr, encore Vérone/Mantoue dans Roméo et Juliette ...

Cette remarque faite, qui oppose a l'hiver moral de la ville les possibilités printanières de la forêt, du jardin, plus tard - avec la Tempête - de l'Ile, revenons au mythe de Pyrame et de Thysbé transfusé dans celui de Roméo et Juliette : tous les mythèmes sont répétés : I, la séparation, c'est celle implacable de"l'antique hargne" (I. Prologue) des Capulet et des Montaigue - comme l'écrit le traducteur P. S. Jouve - II, le tombeau des Capulet où se dénouera le drame - III, l'objet trompeur est ici multipliè : la lettre qui n'est pas parvenue à Roméo, le breuvage léthargique administré à Juliette par frère Laurent - IV, la mort de Roméo, trompé par l'aspect cadavérique de Juliette - V, Juliette se poignardant sur le corps de Roméo comme Thysbé sur celui de Pyrame ... 
Et cette rapide mythocritique de Thysbé/Juliette nous permet de mieux comprendre le mythe que cachait le simple complexe d'Ophélie dans le chef d'oeuvre de ce théâtre - selon Hugo qu'est Hamlet en 1600 ... Car une fois les "pâquerettes, les coucous" écartés et l'eau funèbre séchée, ce que dévoile le drame d'Ophélie et d'Hamlet, c'est encore le mythe de Pyrame et Thysbé : I, la terrible haine du Prince de Danemark pour l'usurpateur, redoublée de la ferme interdiction que met Polonius Jephté, comme l'appelle Hamlet (II.2) - , lord Chambellan de l'usurpateur et son fils Laërtes, à l'amour de sa fille Ophélie pour le Prince ; II, toute la fin du drame, l'acte V, est obsédée par la tombe de la scène 1 où après un dialogue macabre des fossoyeurs, le corps d'Ophélie est porté en terre et où, dans la fosse même, luttent déjà Hamlet et Laërtes. Là encore les objets de tromperie se multiplient : a la scène 7 de l'acte IV, c'est le complot entre le roi usurpateur et Laërtes pour tuer Hamlet par une pointe de fleuret empoisonnée et par une coupe de poison - IV, ce n'est pas Hamlet qui avale le poison, mais sa propre mère la reine - $\mathrm{V}$, et si Hamlet est bien blessé a mort par la lame empoisonnée, la fougue du combat intervertit les armes, Hamlet alors transperce Laërtes, mais également le roi usurpateur, "complots déroutés qui se retournent contre leurs instigateurs" conclut Horatio. Ici le mythe incarné par Ophélie et Hamlet donne a celui de Pyrame et Thysbé toute sa dignité et son ampleur. Le fameux "être ou ne pas être" que profère de façon incohérente le Prince a la scène 1 de l'acte III, juste après l'aveu honteux du roi :

"La joue plâtrée de la courtisane n'est pas plus laide que mon crime sous son revêtement mensonger"!

approuvant le conseil de Polonius à sa fille :

"Nous méritons souvent le blâme lorsque sous couleur de dévotion et d'action pie nous sucrons le diable lui-même"

et juste avant l'affrontement avec Ophélie, en une rupture feinte en une fausse folie, est en son fond choix entre l'être et le mensonge. Amplification du mythe du "ne pas être" que multiplient mensonges et erreurs dans un engrenage terrifiant qui commence par la haine et finit par l'hécatombe de Polonius, d'Ophélie, de la Reine, de Laërtes, de Claudius, d'Hamlet ... Tragédie des erreurs, enfer où toutes les espérances du printemps sont laissées à la porte d'un éternel hiver des actes gelés des tombes ouvertes - $\mathrm{j}$ 'aurais presque envie, en pensant à Rabelais, de dire "des paroles gelées" : 
"des mots, des mots, des mots" ! Hamlet II sc. 2 ; "Des mots, des mots, de simples mots, rien qui parte du coeur" déplorera plus tard Troïlus (V. 3).

La terrasse, les chambres et les tombes d'Elseneur sont plus désespérées, contre la nature, que ne l'étaient les villes maudites Vérone, Messine, Venise ...

Ce monde de l'erreur et le problème adjacent du choix du non être de l'apparence va encore se préciser, dans les tragédies des années du début du XVII siècle, en problème de la fidélité. La fidélité de Pyrame et de Thysbé, de Roméo et de Juliette, voire d'Hamlet et d'Ophélie était indéfectible. Une seconde dérivation du mythe va être celle où à la constance se substitue l'infidélité ou du moins son apparence.

En ce sens les deux tragédies de 1602 et 1603, Troïlus et Cressida et Othello qui se suivent, se complètent aussi. Troillus et Cressida, "texte informe et sinistre", "cynisme et nihilisme" comme l'écrivent certains critiques ${ }^{15}$ n'est bien, à nos yeux, qu'une ébauche de ce que sera Othello. "Violence y est faite aux valeurs" écrit Fluchère, mais ajoutons : violence faite à toutes les valeurs, ce qui ne sera pas en Desdémone. Cressida c'est une Desdémone réellement infidèle, et comme le dit profondément Henri Fluchère :

"la fidélité est l'aspect sentimental et métaphysique de l'identité",

or Cressida est le paradigme de toute absence d'identité. Elle-même avoue, et justifie ainsi cyniquement l'inconstance de sa conduite :

"le défaut que je trouve en nous (femmes), c'est que l'erreur de nos yeux dirige nos sentiments, et ce que l'erreur conduit doit errer ..." (V. 2)

Et l'infortuné Troïlus, à qui l'on vient de montrer Cressida tombant dans les bras du grec Diomède, dans la plus belle scène de ce drame (acte V. sc.2), ne sait plus ce qui est vérité, ce qui est apparence,

"si je raconte comment ces deux êtres se sont accouplés, ne mentirai-je pas en proclamant une vérité ... ?"

Dilemme terrible : "c'était et ce n'était pas Cressida", où, une fois de plus l'identité, l'être, se dissout douloureusement dans l'apparence, dans "les turpitudes des âmes gouvernées par les yeux", 
comme concluait le cynisme de l'inconstante Cressida. Tout le mécanisme de la jalousie est mis à nu dans ce drame, mais, hélas, Troïlus n'avait que trop raison d'être jaloux !

Il n'en sera pas de même pour Othello ni pour Antoine. Il n'est pas utile, ici, de rappeler l'aveuglement du More de Venise, dont la partie damnée de l'àme est, bien sûr, Iago. Iago est bien l'ignoble sacrificateur de l'honneur d'Othello et du printemps de Desdémone. Car la fidélité de Desdémone est ici intacte, c'est lago qui a machiné l'apparence de l'infidélité. L'objet qui la fixe, qui fixera la précipitation brutale du More, est ce mouchoir de Desdémone que Iago cache dans la chambre de l'infortuné Cassio. Et, comme il se doit, ce sombre drame, où l'aveuglement sur les apparences se joint aux pulsions de la jalousie, se clot aussi par une hécatombe : sur le cadavre de Desdémone étranglée, Othello se poignarde, tandis qu'Emilia, épouse de Iago est frappée à mort par son ignoble mari.

Nous retrouvons bien dans cette terrible tragédie quatre des cinq mythèmes constitutifs du mythe de Thysbé/Juliette/Ophélie : - la séparation des amants que signifie tout l'acte I qui résout la farouche opposition du sénateur Brabantio à l'union de sa fille Desdémone avec Othello - l'objet trompeur n'est autre que le fameux mouchoir de Desdémone que lago récupère à l'acte III (sc.3) - quant aux deux conséquences de "l'apparence" elles existent, mais sont pour ainsi dire interverties : la première conséquence c'est l'assassinat de Desdémone par Othello furieusement abusé, et la seconde c'est le suicide éploré d'Othello à qui, enfin, Emilia mourante a révélé son "erreur" funeste. Ne manque que le mythème de la tombe. Nous allons le retrouver dans le drame d'Antoine et Cléopâtre qui, deux ans plus tard (1606), suit celui d'Othello (1604). Certes Cléopâtre

"ramassée comme un reste sur l'assiette du défunt César" comme lui dit aimablement Antoine dans sa colère (acte III, sc.13), n'a pas exactement le même "printemps" que Juliette, Ophélie ou Desdémone. Mais sa fidélité au si faible Antoine est tout aussi intacte que celle de toutes ces vierges sacrifiées. Comme Othello, l'apparence - de l'envoyé de César/Octave, baisant la main de la reine d'Egypte - déclenche la folle jalousie d'Antoine. Et les quiproquos, dignes de Roméo et Juliette s'enchaînent : à l'acte IV sc.14 c'est la fausse nouvelle de la mort de Cléopâtre colportée par Mardian, Antoine se blesse alors mortellement et 
vient expirer dans les bras de Cléopâtre réfugiée dans le tombeau des Ptolémée où finalement Cléopâtre s'immole grâce au venin des aspics ... le drame nous restitue bien le $5^{\mathrm{e}}$ mythème, le tombeau qui manquait à Othello.

Cette remarque faisant tomber l'accent sur l'importance obsédante de l'image du tombeau, du mourir/dormir qui dans la scène 1 de l'acte III d'Hamlet vient en harmonie avec la fameuse question: "Etre ou ne pas être". Ce qui fait "de chacun de nous un couard" c'est :

"cette crainte de quelque chose après la mort. Cette mystérieuse contrée d'où nul voyageur ne revient".

Cette remarque soulignant l'insistance de la mort et du tombeau, soudain est démentie par les principales œuvres qui suivent à un lustre près la disparition d'Elisabeth et ${ }^{\circ}$ de sa dynastie en 1603. A partir de cet instant les "voyageurs" - et certains sont de grands voyageurs, tels Périclès Prince de Tyr, ou Prospero duc de Milan — reviennent de la "mystérieuse contrée". Mais alors le mythe qui animait jusqu'ici toute l'œuvre, avec pour clef de voûte l'obsession de la tombe ne change-t-il pas radicalement lorsque ce mythème est exorcisé, et que le surnaturel vient servir de refuge suprême a l'ordonnancement de l'être?

Dans Périclès, le premier drame de la série ultime et qui commence par l'évocation d'un inceste, malgré la sempiternelle menace de viol qui pèse sur la pure Marina dans les scènes sordides (acte IV, sc. 1,2,4) où elle est livrée dans un lupanar à un maquereau et sauvée par l'intercession de Diane, le tombeau s'il figure bien à l'acte IV (sc.4) n'est qu'un cénotaphe vide. Comme sera vide le cercueil calfaté de Thaïsa sa mère, comme Périclès lui-même sera sauvé de la tempête mortelle. La tragédie de Périclès s'évapore au souffle de deux - sinon trois - résurrections !

Dans Cymbeline roi de Bretagne, cette sorte de tragique cosi fan tutte, nous trouvons bien sûr à l'acte III (sc.5) l'obsession du viol lorsque Cloten veut violer sa demi sœur Imogène fille du roi, puis une avalanche d' "erreurs" et d' "apparences" qui précipitent l'action : bracelet volé et taches sur le sein gauche entrevues qui attestent faussement de l'infidélité d'Imogène, lettre apportée par Pisano, tromperie grâce aux vêtements du mari d'Imogène qu'a revêtus Cloten (acte IV sc. 1), travesti auquel fait écho celui d'Imogène habillée en homme, enfin l'inévitable tombeau où le corps d'Imogène est enseveli aux côtés de celui, décapité, de Cloten. 
Mais à l'acte $\mathrm{V}$, Imogène réapparaît vivante, ainsi que les deux autres fils de Cymbeline. Là encore, comme dans Périclès le tombeau et la mort sont exorcisés, non plus cette fois par la protection de Diane, mais par celle de Jupiter !

Quant au Conte d'Hiver - et soulignons pour nos inconscients ce titre négateur du printemps, bien que notre conscient sache que cette expression ne signifie platement que "conte de bonne femme", mais comme le dit le petit Mamillius le conteur,

"un conte triste fait mieux l'hiver qu'un conte gai" (II. 1) -

c'est en quelque sorte un Othello hyperbolique, où la jalousie meurtrière atteint chez le vieux roi de Sicile Léontès une violence inégalée. Malgré l'oracle rapporté de Delphes (III.2) qui innocente Hermione, le furieux roi persévère dans sa vengeance. Puis apparaît l'inévitable tombeau (III.2) où Hermione terrassée en venant d'apprendre la mort du petit prince Mamillius, mort de chagrin luimême en apprenant le triste sort de sa mère, sera ensevelie avec son fils. Mais à l'acte V (sc.3) le terrible Léontès tout contrit retrouve sa fille Perdita qu'il avait abandonnée à sa naissance à une mort certaine, et la statue d'Hermione - disparue de la terre depuis seize ans - reprend merveilleusement vie ! Une fois de plus la tombe est exorcisée, une fois de plus le printemps est restitué !

Enfin, survient en 1611 la célèbre Tempête, la "pièce de la restauration d'un ordre perdu" dit fort bien Fluchère, la "fin", le "couronnement" de la quête mythique selon W. Knight ${ }^{16}$. "Prospero est l'ordonnateur souverain de cette restauration". En cela le célèbre drame se différencie du Conte d'Hiver dont les trois premiers actes déchaînent la rupture de l'harmonie et l'émergence du mal. Ici dès la 2 e scène du 1er acte, le héros est au-delà des vicissitudes qu'il a subies à Milan

"par trahison nous fûmes enlevés de là-bas, mais par bénédiction ici transportés".

La situation est quasi-postatomique. Après la catastrophe comme - et mieux! - celle que Rolph Liebermann lit dans le Parsifal de Wagner. L'Ile sauvage est une fois de plus refuge à la vertu contre la ville corrompue. On pourrait affirmer d'elle en inversant un vers célèbre: "on me dit une tombe mais je suis une mère" ! Déjà à Milan, tel Montaigne dans sa librairie, c'est à d'austères occupations que se livrait le Duc : "Pour moi, pauvre homme, ma bibliothèque m'était un assez grand duché" ! (I.2). 
Maintenant après les épreuves, car la pièce commence après les épreuves, le Duc devenu l'initié Prospero, se fait le magicien de la Justice : c'est par ses sortilèges qu'il a amené à sa merci dans son île, son frère usurpateur Antonio et son complice le roi de Naples Alonso. Par ce renversement de la procédure scénique habituelle, le mythe que nous avons suivi à la trace dans toute l'œuvre du grand dramaturge, se gauchit en quelque sorte : les mythèmes qui le constituent s'affaiblissent dès lors que le mythe change de régime. Les amants - Ferdinand et Miranda - ne sont que virtuellement séparés, l'île et ses écueils qu'Alonso pense être le tombeau de son fils Ferdinand, sont une fausse tombe puisque Ferdinand est, en réalité, vivant ; la terrible image du "viol de Lucrèce" n'est réduite qu'a des intentions lubriques du monstre Caliban (I,2 ; III,2); les indices de l'erreur et de l'apparence deviennent les habits clinquants "qui servent de leurre pour attraper ces malandrins" qui ont ourdi une "odieuse conspiration" (IV, 1); la folie elle-même, loin d'être feinte, n'est qu'un des puissants charmes de Prospero. L'exclamation de Miranda à la fin de la pièce, à l'acte $\mathrm{V}$

"O prodige ! Que de belles créatures vois-je ici assemblées, que l'humanité est admirable, $\mathrm{O}$ splendide nouveau monde qui compte de pareils habitants ..."

Splendide nouveau monde ? Monde à l'envers comme le disait ce matin François Laroque ? Certes! Mais aussi nouveau mythe qu'annonçait depuis longtemps, contresignée ici par l'apparition de Junon escortée de Cérès (IV, 1), la Providence bienfaisante de dieux évoqués par le tout puissant Prospero: Diane, Jupiter, Apollon

Ainsi, à l'ombre flottante du frêle complexe d'Ophélie, s'est développé pendant les quelques quinze années de création intense du grand dramaturge élisabethain, un mythe - né de l'obsession du viol de Lucrèce et de l'horreur des "apparences" - dont l'accent tombe toujours, au cours de ses trois grandes variantes, sur le sacrifice - et les sacrificateurs ! - du printemps de la vie, de la pureté, de l'innocence. Ce mythe a pris bien des noms, des noms de couple où à l'innocence du sacrifié faisaient pendant les erreurs, les fantasmes des sacrificateurs: Pyrame, se fiant trop aux apparences, sacrifiant indirectement Thysbé, Roméo trompé par l'apparence entraînant la mort de Juliette éveillée de son sommeil cataleptique, Hamlet par son mensonge et sa folie feinte entraînant Ophélie, le terrible Othello berné par le funeste Iago étranglant de 
ses mains la fidèle Desdémone, Antoine nouveau Pyrame éploré mourant dans les bras de Cléopâtre et abandonnant celle-ci à l'opprobre ou/et à la mort ... Dans tous ces drames l'on retrouve toujours plus ou moins les cinq mythèmes - dont celui du tombeau, de la fosse qui donne une terrible conclusion a ces péripéties - qui expriment les drames nés de toutes les déclinaisons de l'erreur: apparence, mensonge, jalousie ... Enfin les ultimes drames, s'ils gardent bien en les atténuant les structures mythiques de tous les autres, bifurquent pour ainsi dire lorsque le mythème du tombeau est comme exorcisé, transcendé par l'intervention surnaturelle de Diane (Périclès), de Jupiter (Cymbeline), d'Appolon (Le Conte $d$ 'hiver) et même - si l'Ile était bien tombeau pour les naufragés du navire fracassé - Junon qu'invoque l'initié Prospéro ( $L a$ Tempête). Lorsque dans un mythe, un mythème essentiel défaille, il y a progressif changement de mythe ...

Notre propos et nos compétences ne sont pas, ici, de chercher les causes intrinsèques (dans la biographie de l'auteur, par une "psychocritique") ou extrinsèques (dans la répercussion imaginaire des événements qui marquent la fin de la dynastie Tudor, par une étude de "mythanalyse") de ce changement de mythe. Nous ne voulions qu'esquisser, en amateur des drames shakespeariens, un mythocritique rapide des principales œuvres du génial poète et dramaturge anglais, souligner combien à travers les grands accents de son génie, Le Songe, Roméo, Hamlet, Othello, Antoine et Cléopâtre, au-delà du touchant "complexe d'Ophélie" un mythe obsédant, celui du "Printemps sacrifié", comme une énigme terrible du désordre engendré par l'erreur, a constamment inspiré l'immense dramaturgie du poète élisabethain. Il serait fructueux d'amplifier cette enquête en examinant à la même lumière les "tragédies historiques" ... Notons toutefois que la réponse célèbre de Prospero (à l'acte IV, 1 de La Tempête de 1610 ...), profession de foi en l'évanescence du monde, inspirée peut-être par Pindare et Eschyle :

"... le monde se dissoudra, comme ce cortège insubstantiel (des nymphes et divinités) s'est évanoui. Nous sommes faits de la même étoffe que les songes, et notre petite vie, un somme la parachève ..."

s'adresse bien plus qu'à Ferdinand, par delà les ans à la non moins célèbre méditation d'Hamlet (III, 1) de 1600 :

"Mourir, dormir, c'est tout! Mourir, dormir, rêver peutêtre. C'est là le hic ! Car échappés des liens charnels, si dans le 
sommeil du trépas, il nous vient des songes ... !! Halte là ! Une telle considération perpétue les calamités de la vie ... !"

La réponse de Prospero, apaisée constatant - comme le dit un titre de Calderòn - que la vie et le monde ne sont que songes, désamorce l'angoisse d'Hamlet qui craignait que la mort porte encore les réalités de la vie ... Soudain le grand dramaturge couronne le songe et l'imaginaire en l'assimilant à l'étre, à notre être le plus profond. L'angoisse d'Hamlet se résorbe, se résout en la toute puissance du poète. Déjà l'imaginaire est hissé au rang suprême de "roi de nos facultés". Le poète dramaturge, après tant de réticence, découvre enfin - selon le beau titre de Claude G. Dubois - que la profondeur est au fond de l'apparence !

1610 , est-ce donc le moment, lorsque le mythe du cruel sacrifice se dissout dans des interventions de l'au-delà, lorsque les machines de la scène d'opéra prennent le relais des sommaires décors du théâtre parlé, plus puissantes que la tombe et ses spectres, de chanter: "... et redeunt Saturnia regna" ?

\section{Gilbert DURAND}

\section{NOTES}

1. A. Gide, "Avant Propos" à CEuvres Complètes de Shakespeare présentées par H. Fluchère. 2 Vol. La Pléiade, Gallimard, Paris, 1959. C'est à cette édition que nous serons fidèle dans cet exposé.

2. P. Somville,. Art et Symbole à la Renaissance. Soledi, Liège, 1983.

3. G. Bachelard, L'eau et les rêves, essai sur l'imagination de la matière. Corti, Paris, 1942 ; J. Libis, L'eau et la mort. Ed. Univ. de Dijon, 1993.

4. J. Perrin, Les Structures de l'imaginaire shelleyen. P.U.G. Grenoble, 1973.

5. R. Bastide, Anatomie d'André Gide. PUF, Paris, 1972. 1958.

6. Cl. Levi-Strauss, L'anthropologie structurale. Plon, Paris,

7. V. Hugo, William Shakespeare. Flammarion, Paris, 1973.

8. Cl. Levi-Strauss, op. cit.

9. Cao Xueqin, Le rêve dans le Pavillon rouge. Trad. franç. La Pléiade, Gallimard, Paris, 1982. Sun du Shiniang, cf. J. Pimpaneau, 
TABLEAU RÉCAPITU!

Chine, litléralure populaire, chanteurs, conteurs, bateleurs. Ed. Ph. Picquier, Paris, 1991 ; cf. également J. Pimpaneau, Histoire de la littéraure chinoise, p. 362 sq., "Le sentiment de l'Amour". Ed. Ph. Picquier, Paris, 1989.

10. J. Burckhardt, La Civilisation de la Renaissance en Italie. 4 vol., Livre de Poche, Paris, 1958.

11. Cf. G. Dubois, L'imaginaire de la Renaissance. P.U.F., Paris, 1985. - Le Baroque, profondeur de l'apparence. Larousse, Paris, 1973.

12. D. Fernandez, Le Banquet des Anges, l'époque baroque de Rome à Prague. Plon, Paris, 1984.

13. Fr. A. Yates, Astrée, le symbolisme impérial au $X V I^{r}$ siècle. Trad. franç. Belin, Paris, 1984.

14. H. R. Jauss, Pour une esthétique de la reception. Trad. franç. Gallimard, Paris, 1980.

15. J. Maesfield, William Shakespeare, Heinemann, 1954.

16. W. Knight, The Wheel of Fire. 1930.

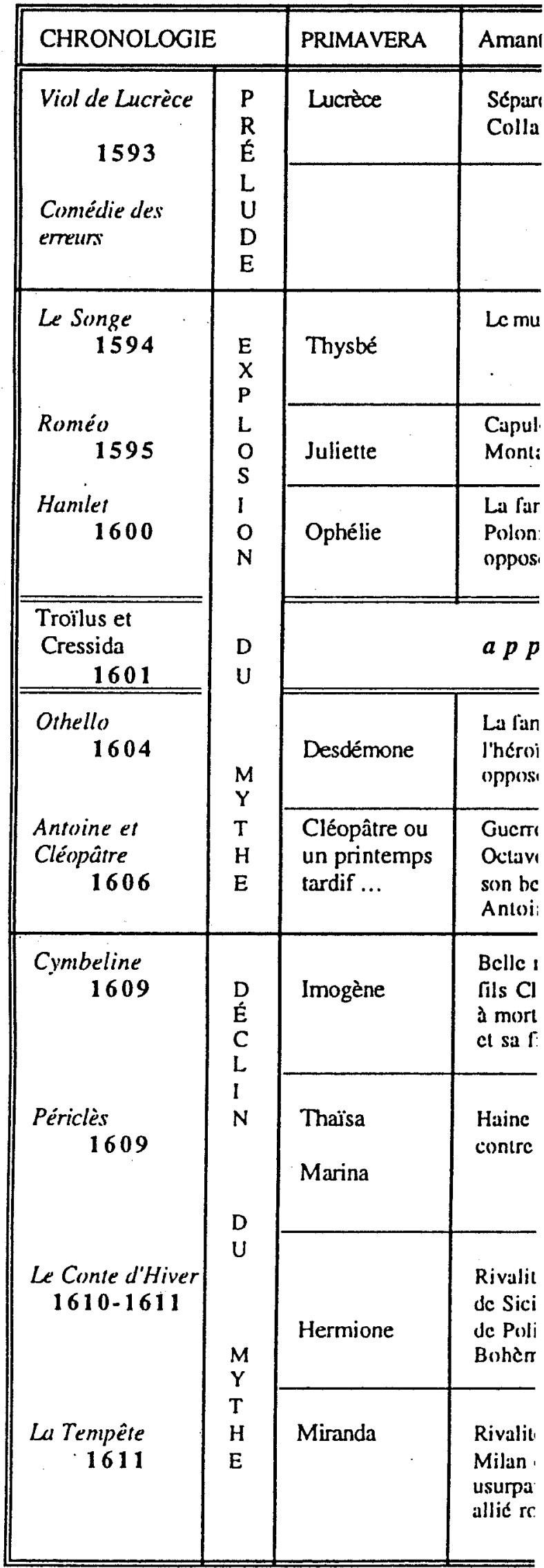


HE DU "PRINTEMPS SACRIFIÉ"

\begin{tabular}{|c|c|c|c|c|}
\hline nces trompeuses & 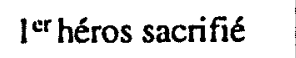 & $2^{\mathfrak{e}}$ héros sacrifié & La Tombe & Résurrection - Rédemption \\
\hline ic de Tarquin & $\begin{array}{l}\text { Vinl el suicide } \\
\text { de Lucrdec }\end{array}$ & & & . \\
\hline \multicolumn{5}{|l|}{ quos des "crrcurs" } \\
\hline $\begin{array}{l}\text { lantille" } \\
\text { glantéc }\end{array}$ & Suicide de Pyrame & $\begin{array}{l}\text { Thyshé se tue sur le } \\
\text { corps de son amant }\end{array}$ & $\begin{array}{l}\text { Tombe de } \\
\text { Ninus }\end{array}$ & \\
\hline gic de Julictle & Suicide de Romćo) & $\begin{array}{l}\text { Julictte sc luc sur le } \\
\text { orps de son amant }\end{array}$ & $\begin{array}{l}\text { Tombe des } \\
\text { Capulel }\end{array}$ & \\
\hline $\begin{array}{l}\text { ic fcinte d'Hamlet } \\
\text { zau trompeur }\end{array}$ & $\begin{array}{l}\text { Folic el suicide } \\
\text { d'Ophélic }\end{array}$ & $\begin{array}{l}\text { Mort d'Hamlet et } \\
\text { hecalombe finalc }\end{array}$ & $\begin{array}{l}\text { Cimcticre et } \\
\text { Tombe } \\
\text { d'Ophélic }\end{array}$ & \\
\hline \multicolumn{4}{|c|}{ 'hème de la jalousie } & \\
\hline $\begin{array}{l}\text { ousic } \\
\text { ibjcl } \\
\text { dchoir }\end{array}$ & $\begin{array}{l}\text { Desdémone } \\
\text { ćloulféc }\end{array}$ & $\begin{array}{l}\text { Suicide } \\
\text { d'Othello }\end{array}$ & & \\
\hline $\begin{array}{l}\text { ic de Cléopâtre } \\
\text { : Octavie } \\
\text { ic d'Antoine envers } \\
\text { isager }\end{array}$ & $\begin{array}{l}\text { Faussc nouvclle de } \\
\text { la mort de Clêpatre } \\
\text { suicide d'Antoine }\end{array}$ & $\begin{array}{l}\text { Suicide de Cléopâtre } \\
\text { morduc par } \\
\text { le serpent }\end{array}$ & $\begin{array}{l}\text { Tombe des } \\
\text { Ptoléméc }\end{array}$ & \\
\hline $\begin{array}{l}\text { ss prcuves d'infidéli- } \\
\text { acclet, jalousie sans } \\
\text { le Posthumus. } \\
\text { stis. }\end{array}$ & $\begin{array}{l}\text { Mort feinte } \\
\text { d'Imogène }\end{array}$ & $\begin{array}{l}\text { Quiproquo, Cloten } \\
\text { travesti en } \\
\text { Posthumus, } \\
\text { décapité }\end{array}$ & $\begin{array}{l}\text { Tombe } \\
\text { d'Imogène } \\
\text { et de Cloten } \\
\text { décapité }\end{array}$ & $\begin{array}{l}\text { Intercession de Jupiter } \\
\text { "ressurrection" d'Imogène }\end{array}$ \\
\hline $\begin{array}{l}1 \text { vendue } \\
\text { adonnćc dans } \\
\text { inar }\end{array}$ & $\begin{array}{l}\text { Mort fcinte de } \\
\text { Murina } \\
\text { Mort de Thaïsa }\end{array}$ & $\begin{array}{l}\text { Péricles muet } \\
\text { de desespoir }\end{array}$ & $\begin{array}{l}\text { Cénotaphe } \\
\text { de Marina } \\
\text { Ccreucil de } \\
\text { Thaîsa }\end{array}$ & $\begin{array}{l}\text { Intercession de Diane } \\
\text { Thaïsa ressuscitce de son } \\
\text { ccrcucil }\end{array}$ \\
\hline $\begin{array}{l}\text { ic de Lceontes } \\
\text { s par l'apparence }\end{array}$ & $\begin{array}{l}\text { La fille d'Hermionc } \\
\text { Perdita "jetce aux } \\
\text { corbeaux" }\end{array}$ & $\begin{array}{l}\text { Mort de l'enfant } \\
\text { Manillius }\end{array}$ & $\begin{array}{l}\text { Tombe du pe- } \\
\text { lit Manillius } \\
\text { ct de sa mère } \\
\text { Hermione }\end{array}$ & $\begin{array}{l}\text { Oracic d'Apollon } \\
\text { vie renduc à la statue } \\
\text { d'Hermione }\end{array}$ \\
\hline $\begin{array}{l}\text { bits clinquants } \\
\ell \text { de lcurre pour } \\
\text { ir les conspiratcurs }\end{array}$ & $\begin{array}{l}\text { Les tribulations du du } \\
\text { ont précédé la pièce }\end{array}$ & IC de Milan & $\begin{array}{l}\text { L'île } \\
\text { "tombeau" } \\
\text { supposé de } \\
\text { Ferdinand }\end{array}$ & $\begin{array}{l}\text { Junon et Cérès au service de } \\
\text { Proserpine retablissent ordre } \\
\text { ct paix }\end{array}$ \\
\hline
\end{tabular}

